\title{
Hosting International Nursing Students through the Lens of Primary Health Care
}

\author{
Karyn Taplay $^{1}$, Jane Moore ${ }^{2, *}$, Michelle Richardson ${ }^{2}$ \\ ${ }^{1}$ Brock University 500 Glenridge Ave.St Catharines, Ontario L2S 3A1.Office: EA 321 \\ ${ }^{2}$ Department of Nursing, Faculty of Applied Health Sciences, Brock University, St. Catharines, Ontario, Canada \\ *Corresponding Author: jane.moore@brocku.ca
}

Copyright (C) 2013 Horizon Research Publishing All rights reserved.

\begin{abstract}
Internationalization is an objective of many academic institutions and programs. Hosting international students was one way to raise the international profile of a nursing program and the institution. In order to plan, implement and evaluate the experience of hosting exchange students the nursing faculty used the principles of primary health care (PHC), which are the fundamental tenants of the program. Although the principles of PHC focus primarily on the health of a client or population they served well as a framework for hosting international students and provided the structure needed to facilitate a student exchange program that could be sustained between an Austrian and a Canadian nursing program.
\end{abstract}

Keywords Internationalization, Nursing Students, Primary Health Care Principles

\section{Introduction}

Internationalization serves as a key marker of a university's prestige, as it assists in facilitating and strengthening international academic linkages [1]. It involves the integration of a global perspective into the teaching, learning, research and community service [2]. At one Canadian mid-sized university, internationalization is considered a critical component of the mission statement that guides the development and administration of programs to facilitate universal understanding and respect through international learning [2]. The faculty at this university are encouraged to use innovative ideas to fulfill the objectives of the mission statement. International courses, faculty research presentations, and the creation of a large international center with a diverse student population have been used to promote internationalization. The nursing department proposed hosting nursing students from another country as a creative strategy to meet the international expectations of the university. This paper reports on the experience of one Canadian nursing program hosting three Austrian nursing students in 2012. The principles of primary health care were used as a framework to plan and implement this exchange experience and will guide the ensuing discussion. Benefits and challenges will be described and recommendations to enhance the international experience will be offered.

\section{Background}

The initiative started with a university in Salzburg Austria approaching Brock University about forming a mutually beneficial relationship towards the goal of internationalization. Brock International is a department within the university focused on developing, facilitating, and strengthening international partnerships [2]. Brock International informed the Nursing Department about the potential to host Austrian nursing students. The Brock University nursing program is similar to the Austrian nursing program as both are four years in length, have students attend classes on campus, and participate in clinical placements in both the hospital and community setting. Similarly, both Canadian and Austrian students undergo a range of clinical experiences from medical, surgical, maternal-child, mental health, and pre-graduate specialty placements. As both nursing programs offer a baccalaureate degree and expressed interest, a memorandum of understanding was initiated. Once in place, the Austrian students were able to register for this experience and receive an elective credit at their university. Upon confirmation of their participation, students were required to pay an administration processing fee of four hundred dollars to Brock International.

The first Austrian students to take part in the exchange included one male and two females in their third year of a four year program. The four week exchange occurred in February 2012. Brock International services arranged the housing and transportation to and from the airport for the visiting students. The overall cost of the exchange was approximately $\$ 3000$ (Canadian) per student and was covered entirely by the Austrian students. Two Brock nursing faculty and three nursing students volunteered to plan and host the Austrian nursing student exchange. 


\section{Planning Using the Principles of Primary Health Care}

The planning of the exchange was guided by principles of primary health care (PHC). The PHC principles were chosen because they reflect the keystone concepts of the Brock nursing program that support all aspects of the curriculum [3]. Using the PHC principles as a framework helped the host faculty be mindful of the complexities and multifaceted factors that are involved in international student exchanges. The five PHC principles are based on the definition by the World Health Organization (WHO) (1978) and include: public participation, accessibility, intersectoral and interdisciplinary cooperation, access to appropriate technology, and health promotion and illness prevention [4].

\section{Individual and Community Participation}

This principle refers to individual decision making and the active role of participation in making personal decisions [4]. Typically it relates to health but in this case we interpreted it to mean the Austrian students should participate in decisions that would meet their educational learning objectives. The initial learning objective that the Austrian students identified was to explore the health of Canadians and the health care delivery models. Understanding the student's goal allowed us to plan. A calendar was created outlining possible class and clinical experiences that the students could participate in. The students made their choices to meet their learning objectives and then individual calendars were made before the students arrived in Canada. Encouraging the students to have an active role ensured that their needs were met. This level of participation allowed for effective and strategic planning. Upon arrival additional learning opportunities in the community were offered such as participating in a head injury prevention education program in the region and attending a midwife clinic. The visiting students had a variety of choice in terms of classes and clinical experiences as well as university activities. The schedule was flexible, allowing for changes if need be. This worked well for the students as they were able to modify and further develop their learning needs once they were in Canada.

\section{Accessibility to Clinical and Health Care Services}

This primary health care principle refers to the accessibility of health care and available health services [4]. Typically the context of accessibility relates to patients but for our purposes we interpreted this principle in our planning to provide similar if not the same access of clinical and health care experiences to the Austrian students that are available to our Canadian students. In order to do this we contacted the clinical placement coordinator within the local health care system. The Austrian students were required to provide documentation of immunization records similar to what is required of Brock students. Once this was in place the students had equal access to health care settings, including the local hospitals and clinics. While in Canada the visiting students had clinical experiences that included: medical, surgical, rehab, and community settings. This provided the Austrian students with opportunities for critical thinking and observed practice experiences. Students also participated in pre and post conference discussions and presentations by professional interdisciplinary staff and took full advantage of the learning opportunities that were available.

\section{Intersectoral and Interdisciplinary Cooperation}

This principle relates to collaboration among health care professionals and includes collaboration across all sectors [4]. Our interpretation of this principle included collaborating with experts in various areas to organize, coordinate, and support a successful experience for the students. In addition to international services, Austrian university staff, and the local health care system, collaborative links with host students, and nursing faculty in Canada needed to be in place and contributed significantly to the success of this experience. The host students at Brock started collaborating with the international students' several weeks before the arrival date via email and social networks. The Canadian students offered suggestions and ideas to help the visiting students to reach their academic and non- academic goals. Collaboration with the nursing faculty was also essential as this provided the visiting students opportunities to attend and be active participants in classes. The faculty welcomed the visiting students into their classes, were available for questions, and offered support related to completion of an academic paper the Austrian students had to write as part of their course objectives. The topic of the paper was a comparison of the Austrian and Canadian health care systems and the role of Registered Nurses in those systems.

\section{Access to Technology}

This principle has three components, first, health care systems are constantly changing and technology is evolving. Second, knowledge exchange of information and research is ongoing and third, continued competence and professional development is essential [4]. Our interpretation of this PHC principle to guide us in hosting international students was also three fold. Having the technology available to the Austrian students for the primary purposes of communication and coordination was essential. The process started with email to facilitate communication and scheduling. Additionally, the students were connected to the library services at Brock and were given full access to internet services. This aided in the student's ability to complete their required coursework while in Canada. The host students felt this principle of PHC was essential as most of their communication with the visiting students was via email or social media.

Our interpretation of the second component of this principle, knowledge exchange, focused on the shared learning about the health care delivery models in Austrian and Canada. When the students arrived one of the host faculty members provided a synopsis of the health care system in Canada. The students also received additional information by attending classes, clinical, and having our 
student handbook available to them. To promote reciprocal learning the students were asked to give a lecture about the health care system in Austria to our nursing faculty and students. Similarities and differences between the health care systems were discussed and there was significant sharing and exchange of knowledge related to health care systems in the two countries. This contributed to the third component of this PHC principle; the need for ongoing competence and professional development. The profession of nursing is evolving, becoming more globalized, and where the provision of care is becoming more complex, dynamic and borderless [5]. Using this PHC principle as a guiding factor when hosting international students, we planned activities on and off campus to introduce the students to the local culture, and in turn increase their cultural sensitivity. On campus activities included: a music concert and intramural sports. Off campus activities included: independent and accompanied travel across Southwestern Ontario and New York State, shopping in Toronto, Niagara Falls and New York City, local wine tours and maple syrup festivals. The Canadian students, through discussions with the Austrian students, had the opportunity to learn about the culture and customs of Austria. This contributed to learning that facilitated professional development in the area of cultural competence and cultural diversity. Using the three components of the PHC principle access to technology contributed immensely to the success of this experience.

\section{Health Promotion and Illness Prevention}

This principle pertains to the health and wellbeing of individuals. It incorporates the determinants of health and focuses on education as well as health promotion activities to prevent illness and maintain health [4]. This principle guided us when planning; we were cognizant of the students' health and wellbeing. Considerations were made regarding scheduling, safety, and illness prevention. When planning the students schedule we made sure to allow a day of rest after travelling to Canada and prior to returning to Austria. We felt this was essential to mitigate any negative effects of jet lag and the time change. This allowed the students to feel rested and prepared to meet their learning objectives. Additionally the scheduling of events was flexible; students had multiple opportunities throughout their stay to meet their learning needs. In order to promote safety for the visiting students personal and professional contact information was provided so they had a contact person available during their entire time in Canada. We felt that this fostered their independence while providing a safety net if needed. Additionally, the host students provided their contact information and often accompanied the Austria students during both on and off campus activities.

Illness prevention was also a consideration while planning this experience. If the students were to fall ill during their stay Brock International ensured the students would have access to health care services. The visiting students were enrolled in the University Health Insurance Plan (UHIP). The cost was minimal to the student (\$57 Canadian per month) [6]. This provided the student with comprehensive health care coverage while at the university and included: medical, dental, and pharmaceutical services. This principle of PHC served us well in the planning of this exchange experience. The Austrian students remained healthy and were able to fully enjoy and benefit from their experience.

\section{Benefits and Challenges}

The Austrian student exchange provided numerous benefits for the visiting students, host faculty and students. These benefits included an increased knowledge of cultural diversity and globalization of nursing, and a better understanding of the social, political and economic factors that impact health care in Austria and Canada. It is important for nursing students to learn about these factors to broaden their horizons and gain different perspectives. An additional benefit of knowledge exchange is the building of professional relationships. This is significant to both faculty and nursing students. The exchange provided faculty with potential research and scholarship opportunities. To date, one faculty member has visited the Austrian University and presented her research on collaboration among nurses. In addition, building professional relationships among nursing faculty and students, allowed them to develop a broader network of nursing colleagues and peers.

While the benefits were numerous there were also challenges. Although accessibility to clinical experiences were equal to that of our students there were two limiting factors beyond the control of the university that did impact the students experience. The first was transportation as students were limited to certain clinical sites due to the lack of public transportation across the region. This resulted in the host faculty/students organizing private transportation. This proved to be extremely difficult given limited access to vehicles among the Canadian nursing students. Anticipating this challenge in the future would enable us to plan car-pooling in advance or seek assistance from Brock international for transportation. The second challenge, Austrian students were only permitted observational experiences in clinical as mandated by the local health care system therefore were unable to provide any direct patient care; although disappointed, thestudents expressed understanding. As this exchange program develops perhaps this issue can be revisited with the local health care system. Another challenge identified by the Austrian students was the limited healthy food available on and off campus. The students commented that they were "amazed at the amount of treats" (cookies and donuts) that were readily available and inexpensive. Recognizing this challenge will help us in the future to use the PHC principle of health promotion and illness prevention more extensively to consider the overall health of the students and be more cognizant of nutritional aspects. 


\section{Conclusion}

In the future we plan on continuing to offer an international exchange program between the two nursing programs. Already, we have an additional four Austrian students signed up to participate in 2013-2014. Ideally the future includes organizing a similar exchange for Brock students to visit the Austrian nursing school as interest has already been expressed. Hosting the international students provided an exciting experience for everyone involved both in Canada and Austria. The principles of PHC worked extremely well as a guide to hosting the international nursing students, it provided an excellent framework that facilitated this experience. Our interpretation of the intricacies and complexities that are inherent within the principles of PHC provided us the opportunity to look at this experience from a holistic perspective, guided our thinking, planning, and organizing, and contributed to the success of this experience.

\section{REFERENCES}

[1] Lee, J. (2010). International students' experiences and attitudes at a US host institution: Self reports and future recommendations. Journal of Research in International Education, 9, 66-84. doi: 10.1177/1475240909356382

[2] Brock University. (2012a). Mission and Vision Statements. Retrieved from Retrieved from http://www.brocku.ca/research/about-brock-research/mission -vision.

[3] Brock University. (2012b). Department of Nursing, Philosophy of Nursing. Retrieved from http://edit.brocku.ca/webfm_send/2215.

[4] Canadian Nurses Association (CNA). (2002). Primary Health Care: A new approach to health care reform. Retrieved from http://www2.cna-aiic.ca/cna/documents/pdf/publications/phc presentation_kirby_6602_e.pdf.

[5] Stamler, L, L. \& Yiu, L. (2012). Community Health Nursing: A Canadian Perspective, 3rd ed. Toronto: Pearson.

[6] Brock University. (2012c). The University Health Insurance Plan. Retrieved fromhttp://www.uhip.ca/_uploads/en_PremiumTable.htm 